\title{
Normal Vestibulo Ocular Reflex (VOR) gain Measured Using the Video Head Impulse Test (vHIT) in Healthy Young Adults
}

Jamaluddin $S A^{a}$, Omar $N A^{a}$

${ }^{a}$ Department of Audiology and Speech Language Pathology, Kulliyyah of Allied Health Sciences, International Islamic University Malaysia

\section{ABSTRACT}

Introduction: Many new objective tests to assess the function of specific structures of the vestibular organ are currently adopted in vestibular clinics. One of the objective assessments include the video head impulse test (VHIT) where gain \& velocity responses of eye relative to the head movements are recorded using an infrared camera. Methods: Thirty normal hearing subjects age between 18 to 25 years old participated in this study. At least ten Lateral, Left Anterior Right Posterior (LARP), and Right Anterior Left Posterior (RALP) responses were recorded for each participant by making small and rapid unpredictable head movements. Results: The average velocity gain for Lateral responses at $40 \mathrm{~ms}, 60 \mathrm{~ms}$ and $80 \mathrm{~ms}$ were $1.05 \pm$ $0.003,1.03 \pm 0.002$ and $1.01 \pm 0.003$ respectively. The LARP average velocity regression were $1.01 \pm 0.24$ for Left Anterior and $1.05 \pm 0.25$ for Right Posterior, with an average gain asymmetry of $5.13 \%$. The RALP average velocity regression were $1.08 \pm 0.31$ for Right Anterior and $1.12 \pm 0.30$ for Left Posterior, with an average gain asymmetry of $5.87 \%$. One sample T-test were conducted to compare Lateral responses to a previous study by Mossman et al. (2015) where significant differences in velocity gain at $60 \mathrm{~ms}$ and $80 \mathrm{~ms}$ between studies were found where, $\mathrm{t}(59)=5.56, \mathrm{p}<0.01$ and $\mathrm{t}(59)=2.86, \mathrm{p}<0.01$ respectively. Conclusion: This indicates the importance of establishing on-site norms for every clinical settings as techniques used and equipment differences could affect the results.

KEYWORDS: Video Head Impulse Test, Vestibulo-ocular reflex, Semicircular canal, Saccades, Vertigo.

\section{INTRODUCTION}

Human ear consists of three sections named as the outer, middle and inner ear. The inner ear comprises of two main functions which serves as sensory organs for hearing and balance. Three important structures that perform as balance sensory end organs are known as the utricle, saccule and three semicircular canals where they help to regulate our sense of linear and angular accelerations with reference to the head position and gravity. Sensation of linear acceleration is supportive upward, downward, forward and

Corresponding author:

Nurul Amira Omar

Department of Audiology and Speech Language

Pathology,

Kulliyyah of Allied Health Sciences,

International Islamic University Malaysia,

25200 Kuantan, Pahang, Malaysia.

Tel No: +6012-4915145

Email: amira5900@gmail.com backward movements is regulated by the utricle and saccule and the semicircular canals on the other hand regulate angular movement which refers to rotational movements of the head. The three semicircular canals are known as horizontal semicircular canal (HSCC), anterior semicircular canal (ASCC) and posterior semicircular canal (PSCC), respectively. They are located in both right and left petrous portion of the temporal bones of our skull. In order for all the output of head movements be combined and processed in the brain, their orientation are in right angles at ninety degrees from one canal to the other canals. If the messages are sent incorrectly to our brain, our bodies will react differently and sensation of dizziness, nausea and general unsteadiness will be apparent. According to Katz, Chasin, English, Hood, \& Tillery, $2015^{1}$ a sudden loss of one vestibular end function will result in vertigo, imbalance, nausea and vomiting. 
We are unable to directly assess these vestibular sensory structures however we can evaluate them using our secondary motor responses such as our voluntary and involuntary reflexes to maintain eye position or postural control during movements for example the vestibulo-ocular reflexes (VOR). The VOR is measured by using the ratio of eye movements in distance and velocity over the head movements. The basic pathway mediating this reflex consists of primary afferent fibres in the vestibular nerve and the secondary vestibular neurons in the vestibular nuclei, which receive synaptic inputs vestibular end organs and project to the motor neurons innervating the extraocular muscles. There are additional central pathways and structures that contribute to the VOR, but the information carried by the axons of secondary vestibular neurons to extraocular moto neurons is essential for the normal operation of the reflex. To produce VOR responses, the vestibular system control the six pair of the eye muscles that is controlled by our oculomotor and abducens nuclei in the middle brainstem. When we turn our head rightward for example, it will stimulate the right horizontal semicircular canal hair cell to depolarize. This excitation process will increase the neuron firing rate at the right vestibular nucleus and the sixth cranial nerve.

The excitation of the sixth cranial nerve neuron will cause the contraction on the left lateral rectus and right medial rectus through excitation of neuron at the third cranial nerve. This contraction will cause the compensatory eye movements to the left side.

A normal VOR system would yield a gain that is equal to 1 which shows that the compensatory eye movements is equal and opposite in direction to the head movements (McCaslin, Jacobson, Bennett, Gruenwald and Green, 2014). ${ }^{5}$ One method of recording the VOR responses is by using the Video Head Impulse Test. The Video Head Impulse test (VHIT) can be done in vestibular clinics to access the integrity of all of the semicircular canal VOR functions individually.

The Head Impulse Test (HIT) was first introduced by Halmagyi and Curthoys in 1988 as a bedside test to identify unilateral peripheral vestibular hypofunction of a patient. It uses a fast, low amplitude head rotations (at angle of 10 to 20 degrees) but with high acceleration (100 degree/s) in the plane of the semicircular canals being tested (Jorns-Häderli, Straumann, \& Palla, 2007). ${ }^{6}$ The tester or clinicians will stand in front of the patients while holding the patient's head. Patients were asked to maintain their gaze normally on the tester's nose or to look straight ahead. A small low amplitude high acceleration head impulses are generated to the left and right side of the patients. In cases of impaired vestibular functions system, the subject's eye will move with the head accelerations and thus, a corrective saccades to re-fixate the gaze back on the tester's nose can be detected after the head thrust. For vestibular deficit patients who have deficits in their VOR functions, the slow phase eye velocity is reduced, which will cause their eye to produce a catch-up eye movements called saccades to re-fixate at the target. These corrective saccades indicate a sign of which side of the preceding head rotations is impaired. However, there are some constraints that limit this technique of its potential. Normally, covert saccades which are saccades that occur during the head thrust will be missed and might not be visible to the naked eye, as compared to overtly visible saccades that can be detected by trained clinicians. As such, only overt saccades can be used as a base for diagnoses of canal paresis. Furthermore, there are no objective measurements of VOR gain and clinicians basically report the findings based on subjective visual observation by reporting the presence or absence of saccades.

To address these issues, the Video Head Impulse Test (VHIT) was developed recently to record and calculate the VOR gain value for the compensatory eye movements. It uses a lightweight, minimal slip, high speed video recording ( $250 \mathrm{~Hz}$ sampling rate) to track the pupil movements during brief, passive and unpredictable head rotations. Subjects are being seated upright in a lit room and positioned 1 metre from the target position at an eye level of the subjects. Typical VOR mediated eye movements exceeds $100 \%$ sec, thus, a high speed, high resolution camera is needed to differentiate the slow and fast eye movements. The VHIT camera is designed to detect changes in the pupil movement while a gyroscope correspondingly will precisely measure the changes of head velocity to allow for direct comparison (Patterson, Bassett, Mollak, \& Honaker, 2015). H. G. MacDougall et al., $2009^{7}$ stated that by only $100^{\circ}-150^{\circ} / \mathrm{sec}$ head velocity acceleration, VHIT can precisely detect a deficit in the semicircular canal functions for a patient which 
only suffers from acute vestibular deficit. The accuracy of the VHIT to detect canal paresis was verified by a study by MacDougall, McGarvie, Halmagyi, Curthoys, \& Weber, $2013^{2}$ by comparing the results obtained with the results from the scleral search coil method which is the current gold standard to detect dysfunctions of the semicircular canal. Theystated that there were no significant difference between using scleral search coil technique as compared to the high speed video recording of head impulse technique in detecting semicircular canals dysfunction. Twelve patients with unilateral, bilateral and individual semicircular canal dysfunction were tested and the gain was compared with seven normal subjects. Horizontal, right anterior left posterior (RALP) and left anterior right posterior (LARP) gains were calculated. Eye movements were recorded simultaneously using a $2 \mathrm{D}$ video head impulse test and 3D scleral search coil and the results showed no significant differences between both measurements. The mean VOR gain difference measured with VHIT and search coil was $0.05 \pm 0.14$ for the LARP plane and $-0.04 \pm$ 0.14 for the RALP plane. The coefficient of determination $R^{2}$ was 0.98 for the LARP plane and 0.98 for the RALP plane. This study suggests that the video procedure accurately detects dysfunctions in the six semicircular canals similar to the scleral search coil technique. More importantly, this allows for an alternative method of accurate VOR measurement especially because the scleral search coil is not suitable to be performed in the clinical settings as it is an invasive procedure and subject's eyes needs to be anaesthetized.

The gain parameters of lateral semicircular canal were measured by using instantaneous velocity gain which measures at specific time interval that is 40 $\mathrm{ms}, 60 \mathrm{~ms}$ and $80 \mathrm{~ms}$. Meanwhile for vertical semicircular canal that is left anterior right posterior (LARP) and right anterior left posterior (RALP), the gain was measured using average velocity regression gain which measures the gain from 1-100 ms time frame which is more specific and sensitive to detect vestibular deficit as it is within the reaction time of the VOR reflexes. According to Mossman, Mossman, Purdie, \& Schneider, 2015, gain for lateral semicircular canal was measured at $60 \mathrm{~ms}$ due to frequent eye catch up saccades seen in some patients with vestibular deficit and at $80 \mathrm{~ms}$, gain measurement is taken in case artefact occurred during the early stages of head rotations. This difference in time recording is very important to detect any abnormalities such as saccades being detected during and after head acceleration and also to check for the reliability of the recording.

VHIT have some limitations and that might cause inconsistent and limited normative data being published for now. Firstly, it could be difficult to deliver accurate and consistent angle for the vertical semicircular canal head rotations, hence skilled and well-practised clinicians is required to perform the manoeuvre. Besides, the physical characteristics of the goggle itself appears to be one form of limitation. There was one study conducted by (Hamish Gavin MacDougall, McGarvie, Halmagyi, Curthoys, \& Weber, 2013b) which stated that the small eyes of Asians will hamper the recording of the eye velocity especially in the vertical semicircular canal. This cause less eye tracing to be recorded by the system. Apart from that, patient conditions also play an important role to obtain a consistent result. Patients with "lazy" eyes and stiff neck muscle might also give a false reading. These inconsistencies could have led to less normative findings being published for vertical semicircular canal. Therefore, clinicians need to be vigilant in making sure that recording error are not based on measurement artefacts due to loss of attention.

There are several published normative values which are used to indicate a VOR deficit in a patient with vestibular hypofunction. The normal range of gains for VHIT that are often referred to for lateral and vertical maneuvers are 0.8-1.2 and 0.7-1.2, respectively (Mossman et al., 2015). According to Hamish Gavin MacDougall, McGarvie, Halmagyi, Curthoys, \& Weber, 2013a, a VOR deficit is defined when the VHIT gain obtained is less than 0.68 with the presence of corrective saccades. Alternatively, findings by Weber, MacDougall, Halmagyi, \& Curthoys, 2009 suggested a peripheral deficit can occur if gain obtained is less than 0.8 for lateral semicircular canal and gain less than 0.7 for LARP and RALP with presence of covert and overt saccades. A few other studies noted some important findings in terms of the outcome of this test as VHIT was found to be affected by age and certain vestibular disorders. Studies by Mossman et al., 20153; Tian, Crane, Wiest, \& Demer, $2002^{6}$ stated that the horizontal vestibulo-ocular reflex velocity gain decreased slightly by 0.012 and 0.017 per 
decade as age increases at $80 \mathrm{~ms}$ and $60 \mathrm{~ms}$, respectively. However, vHIT stands relatively well with age as compared to Vestibular Evoked Myogenic Potential that might be lost due to aging that could deteriorate the saccular and corresponding neural functions.

The use of vHIT has been shown to provide valuable information to investigate peripheral vestibular disorders. Some vestibular disorders such as benign paroxymal positional vertigo, idiopathic vestibulopathy, migraine vestibulopathy, Meniere's disease and acute vestibular attack may affect the VOR gain as these disorders have a direct effect on the endolymphatic fluid movements in the semicircular canals. A recent study done by McGarvie, Curthoys, MacDougall, \& Halmagyi, $(2015)^{7}$ suggested that different physical enlargement of the membranous duct in the hydropic labyrinths could cause different interpretation of results from caloric response and vHIT. In this study they examine the results of 22 subjects with unilateral Meniere's disease by using both VHIT and caloric testing. The data revealed that by using VHIT, the VOR gain tends to show normal results in the affected ear while abnormal irrigation response in caloric testing in the affected ear. This study demonstrates that even though vHIT and caloric response have same dissociation in measuring the horizontal semicircular canals, but in fact, the outcomes are different. The head impulse test measures the eye movements during the horizontal angular accelerations as the same magnitude generated during normal activities.

The caloric response measures the horizontal eye velocity in response to temperature stimulus delivered to the external auditory meatus in stimulating a VOR response, whereby a heat is delivered via air or water to cause activation of hair cells in the horizontal canal. Hydrostatic mechanism changes occur in the horizontal semicircular canal of the irrigated ear due to the thermally-induced density difference between two arms of the horizontal canal, and cause the bending of the cupula and thus deflect the hair cells and resulting in the eye velocity response. This mechanism could explain why there are different responses using different stimuli in testing Meniere's disease patients. Some vestibular disorders such as benign paroxysmal positional vertigo, idiopathic vestibulopathy, migraine vestibulopathy, Meniere's disease and acute vestibular attack may affect the VOR gain as these disorders have a direct effect on the endolymphatic fluid movements in the semicircular canals. As mentioned earlier, the normal VOR gain is equal to 1 , however for patients with vestibular deficits, the gain may be significantly reduced with observable covert and overt saccade which is a clear indication of a localising vestibulopathy. The current guideline in use is that a gain of less than 0.8 is considered abnormal for lateral semicircular canal and gain less than 0.7 for LARP and RALP with presence of covert and overt saccades (Weber, MacDougall, Halmagyi, \& Curthoys, 2009). ${ }^{4}$

This study is therefore conducted mindful of the lack of normative data especially in the vertical semicircular canal (LARP and RALP). It also aims to compare current data with the previous findings by Mossman, Mossman, Purdie, \& Schneider, 2015 as potential references to be used in the vestibular clinics.

\section{METHODS}

\section{Subjects}

Thirty subjects which included 15 male and 15 females participated in this study. Their age ranged between 18 and 25 years old. The distribution of gender for this study were divided equally to avoid any gender bias in case of any gender factors. The exclusion criteria for this study included subjects with head or neck injury, restricted head movements and history of vertigo. Basic routine audiological clinical testing such as otoscopic examination, tympanometry testing and Pure Tone Audiometry testing was done prior to the test as part of participant selection criteria.

\section{Procedure for Video Head Impulse Test (vHIT)}

EyeSeeCam vHIT system version 1.2 (Interacoustics Ltd, Denmark) is a light weight goggle with infrared camera was used to conduct the Video Head Impulse Test (VHIT) to measure the pupil movements in relation to the head movements. Subjects were asked to sit 1.5 metres in front of the fixation target point at eye level. The tester stood behind the subject with three fingers clasped below the mandible of patient and a thumb and forefinger above the jaw line. Ten head thrusts were 
administered in the horizontal plane both to the left and right at a small angle approximately 10-20 degree with unpredictable timing and direction. For LARP and RALP stimulation, the subject's head was moved downward and upward according to the corresponding angle being tested.

\section{RESULTS}

1. Instantaneous Velocity Gain at $40 \mathrm{~ms}, 60 \mathrm{~ms}$ and $80 \mathrm{~ms}$ for lateral semicircular canal functions bilaterally

The average instantaneous velocity gain for $40 \mathrm{~ms}$, $60 \mathrm{~ms}$ and $80 \mathrm{~ms}$ were $1.05 \pm 0.003,1.03 \pm 0.002$ and $1.01 \pm 0.003$, respectively. This result showed consistent responses where the gain of the head to eye velocity is essentially equal to 1 which indicates that whenever head rotated in the horizontal plane, the eye would follow the head rotations by the same amount of distance and acceleration. It is also important to note that the lateral semicircular canal stimulation using vHIT is easy to perform as the plane of head rotations is not difficult to be repeated. Based on this study, we also found that the lateral manoeuvres yielded consistent results as the overall standard deviation of responses are small with no significant difference between right and left ears.

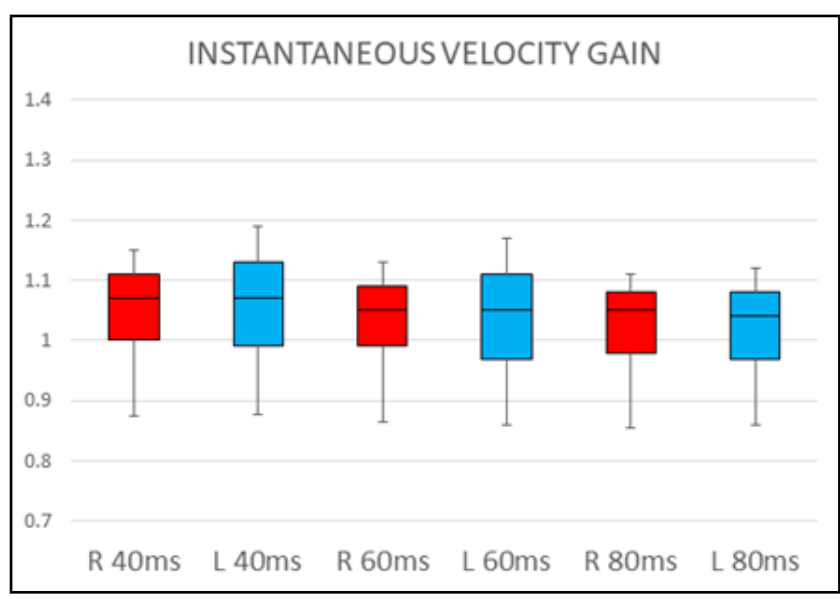

Figure 1: Average Instantaneous Velocity Gain measured at $40 \mathrm{~ms}, 60 \mathrm{~ms}$ and $80 \mathrm{~ms}$ for left and right ears

2. Comparison of instantaneous Velocity Gain at $40 \mathrm{~ms}, 60 \mathrm{~ms}$ and $80 \mathrm{~ms}$ with a previous study

The mean obtained for $60 \mathrm{~ms}$ and $80 \mathrm{~ms}$ was compared with the previous study done by (Mossman et al., 2015) $)^{3}$ using one sample t-test. The results show that there were significant differences in the instantaneous velocity gain where, $\mathrm{t}(59)=5.56, p<0.01$ and $\mathrm{t}(59)=2.86, p<$ 0.01 obtained at 60 and $80 \mathrm{~ms}$, respectively. However, the gain at 40 ms was not compared as no value were provided in the article.

Table 1: Overview differences between current study and Mossman's study

\begin{tabular}{lcc}
\hline Ttem & Current Study & $\begin{array}{c}\text { Mossman et al., } \\
\text { 2015 STUDY }\end{array}$ \\
\hline Sample Size & 30 young adults & $\begin{array}{c}60 \text { control } \\
\text { subjects }\end{array}$ \\
Age & 20 to 30 years old & 20 to 80 years old \\
Equipment & EyeSeeCam vHIT & EyeSeeCam vHIT \\
\hline
\end{tabular}

3. Measurement of the Average Regression Velocity for Left Anterior Right Posterior (LARP) and Right Anterior Left Posterior (RALP)

By using paired sample t-test, the LARP average velocity regression gains were not statistically different from RALP average velocity regression gain as the $p$-value for LARP was 0.083 and 0.127 for RALP.

Table 2: Comparison of the average velocity regression for Left Anterior Right Posterior and Right Anterior Left Posterior

\begin{tabular}{cccc}
\hline $\begin{array}{c}\text { Left Anterior Right } \\
\text { Posterior (LARP) }\end{array}$ & \multicolumn{2}{c}{$\begin{array}{c}\text { Right Anterior Left } \\
\text { Posterior (RALP) }\end{array}$} \\
\hline $\begin{array}{c}\text { Left } \\
\text { velocity } \\
\text { regression }\end{array}$ & $\begin{array}{c}\text { Right } \\
\text { velocity } \\
\text { regression }\end{array}$ & $\begin{array}{c}\text { Right } \\
\text { velocity } \\
\text { regression }\end{array}$ & $\begin{array}{c}\text { Left } \\
\text { velocity } \\
\text { regression }\end{array}$ \\
\hline 1.01 & 1.05 & 1.08 & 1.12
\end{tabular}

Gain asymmetry: $5.13 \%$

Gain asymmetry $5.87 \%$

\section{DISCUSSION}

Instantaneous Velocity Gain at $40 \mathrm{~ms}, 60 \mathrm{~ms}$ and $80 \mathrm{~ms}$ for lateral semicircular canal functions bilaterally

This study revealed that the lateral manoeuvres yielded consistent results with no significant differences between the left and right ears. This is mostly due to the fact that lateral semicircular canal head impulse stimulation are easier to perform and the plane of head rotations is easily referenced. The results are as expected for participants with 
supposedly normal balance function as consistent responses were obtained where the gain of the head to eye velocity is equal at which the eye moves in the opposite direction at equal velocity and gain.

Instantaneous Velocity Gain at $40 \mathrm{~ms}, 60 \mathrm{~ms}$ and 80 ms with a previous study

Comparison between our current studies with Mossman et al., $2015^{3}$ showed a significant difference due to a couple of factors. The differences could be attributed to the design of the video goggles whereby the circumference of the googles does not seem to fit participants' eyes. Some participants' complain of pain on pressure points at the lateral wall of the orbit of the eye particularly at the zygomatic process of the frontal bone. We also encountered some difficulty in getting clear visualization of the pupil as the goggles were squeezing the eyelids. To overcome this problem, the tester had to adjust the position of eyelids to ensure the pupil are easier to trace. Additionally, the camera that was attached at the goggle were quite heavy and could cause inertia during the head movements as the camera rocks from front to back more easily than side to side. This is especially prevalent for the vertical canal manoeuvres. This effect can be classified as a google slippage problem which can produce artefact similar to the ones encountered during the recording as reported by Weber et al., 2009 ${ }^{4}$. In order to avoid this problem, the goggle had to be tighten more which increased the chances of pain. During this study, some artefacts were identified because a few participants did not focus on the fixation point. This caused the camera to incorrectly trace the pupil movements during the head rotations and caused inconsistency eye recordings. In order to avoid this, it is suggested that the room lighting is slightly dimmed and the fixation point is illuminated in some ways. Additionally, the tester should be vigilant in ensuring that the participant's eyes remain fixated to the reference point.

\section{Measurement of the Average Regression Velocity for Left Anterior Right Posterior (LARP) and Right Anterior Left Posterior (RALP)}

For the gain measured at the vertical canal for both LARP and RALP, it can be seen the gains obtained here were quite low as compared to lateral manoeuvre. This might be due to the plane of the vertical semicircular canal that is more difficult to be delivered accurately and thus, more eye tracings are rejected by the vHIT system. In order to address this issue, adequate training should be given to the clinician to ensure consistent head thrust can be made. Additionally, it is also helpful to constantly remind participants to relax their neck muscles and take adequate breaks between head thrusts.

\section{CONCLUSION}

The importance of this research findings serves as a clinical benchmark for subjects with normal hearing and balance function. Further validation of this data could help to establish a normative data to be used as a clinical reference by comparing current data with VOR gain of subjects with confirmed peripheral vestibular loss. This study also clearly showed that the lateral manoeuvres yielded more consistent results as they were easier to be executed on both sides of stimulation. This is different for the vertical manoeuvres where the plane of the head rotations were more difficult to be delivered and hence explains why limited data is published in the literature. Finally, the values obtained were different compared to the study by Mossman et al. (2015). This suggests that on-site norms are important especially when there is variability in equipment and setting.

\section{REFERENCES}

1. Katz, J, Chasin, M, English, K, Hood, L, \& Tillery, K. Handbook of Clinical Audiology. Handbook of Clinical Audiology. 2015: 381-397.

2. MacDougall, HG, McGarvie, LA, Halmagyi, GM, Curthoys, IS, \& Weber, KP. "Application of The Video Head Impulse Test To Detect Vertical Semicircular Canal Dysfuction.” Otology \& Neurotology : Official Publication of the American Otological Society, American Neurotology Society [and] European Academy of Otology and Neurotology, (2013) 34(6), 974-9.

3. Mossman, B, Mossman, S, Purdie, G, \& Schneider, E. "Age dependent normal horizontal VOR gain of head impulse test as measured with video-oculography." Journal of Otolaryngology Head and Neck Surgery. Journal of Otolaryngology - Head and Neck Surgery, (2015) 44(July)

4. Weber, KP, MacDougall, HG, Halmagyi, GM, \& 
Curthoys, IS. Impulsive testing of semicircularcanal function using video-oculography. In Annals of the New York Academy of Sciences (2009) (Vol. 1164, pp. 486-491).

5. McCaslin, DL, Jacobson, GP, Bennett, ML, Gruenwald, JM, \& Green, AP. Predictive properties of the video head impulse test: measures of caloric symmetry and self-report dizziness handicap. Ear and Hearing, (2014) 35 (5), e185-e191.

6. Tian, JR, Crane, BT, Wiest, G, \& Demer, JL. Effect of aging on the human initial interaural linear vestibulo-ocular reflex. Experimental Brain Research, (2002) 145(2), 142-149.

7. McGarvie LA, Curthoys IS, MacDougall HG, \& Halmagyi GM. What does the dissociation between the results of video head impulse versus caloric testing reveal about the vestibular dysfunction in Ménière's disease? Acta OtoLaryngologica, (2015)135(9), 859-865. 